\title{
Effect of Packing and Storage Methods on Quality and Shelf Life of Onion (Allium cepa L. var. Aggregatum Don.)
}

\author{
V. Anbukkarasi*, P. Paramaguru, L. Pugalendhi and P. Jayakumar \\ Horticultural College and Research Institute, Tamil Nadu Agricultural University, \\ Coimbatore, India \\ *Corresponding author
}

\section{Keywords \\ $\mathrm{CO}(\mathrm{ON}) 5$, Onion, Packing and storage methods, Quality and storage life \\ Article Info \\ Accepted: \\ 07 October 2020 \\ Available Online: \\ 10 November 2020}

\section{A B S T R A C T}

In India, presently about 35 to 40 per cent of the onion is estimated to be lost as postharvest losses during various operations including handling and storage. Serious losses occur during storage due to sprouting and rotting. Keeping this in view, an investigation was carried out to study the effect of different packing and storage methods on storage life of aggregatum onion cv. CO on 5. This experiment was conducted at Department of Vegetable Crops, Horticultural College and Research Institute, Tamil Nadu Agricultural University, Coimbatore during 2008-2010 and laid out in factorial completely randomized design (FCRD) with eight treatments and three replications. Pre-harvest spray with growth inhibitors and fungicides were given. The pre-harvest sprayed onion bulbs were harvested, cured and subjected to different packing and storage methods. Among the different packing and storage treatments, the lowest physiological loss in weight $(5.18 \%)$, sprouting $(0.62 \%)$, rotting $(0.64 \%)$, rooting $(0.00 \%)$, total loss $(6.78$ per cent $)$ and quality parameters viz., TSS (17.22 ${ }^{\circ}$ Brix), ascorbic acid (10.24 mg $\left.100 \mathrm{~g}^{-1}\right)$, pyruvic acid $(2.53$ $\left.\mu \mathrm{mol} \mathrm{g}^{-1}\right)$, sulphur content $(0.704 \%)$, total phenolics content $\left(625.56 \mu \mathrm{g} \mathrm{g} \mathrm{g}^{-1}\right)$, phenylalanine ammonia lyase activity $\left(6.86 \mu \mathrm{g} \mathrm{ml}^{-1} \mathrm{~min}^{-1}\right)$, peroxidase activity (1.825 absorbance $\mathrm{g}^{-1} \mathrm{~min}^{-1}$ ) and polyphenol oxidase activity (1.321 absorbance $\mathrm{g}^{-1} \mathrm{~min}^{-1}$ ) were observed and bulbs in ventilated room temperature storage method. The harvested bulbs stored under low cost bottom ventilated storage structure realized maximum shelf life (up to 6 months) compared to spreading of bulbs in room temperature.

\section{Introduction}

Onion is one of the potential foreign exchange earners and is one among the vegetables, where India figures prominently in the world's export market. India stands first in production sharing eight per cent of the world production. During 2018-19, onion is cultivated in an area of 21.85 lakh ha with the production of 232.62 lakh tons (National Horticultural Board, 2019). The major Onion producing states are Maharashtra, Madhya Pradesh, Karnataka, Bihar, Rajasthan, Andhra Pradesh, Haryana, West Bengal, Gujarat and Uttar Pradesh in the country. These States account for almost 90 per cent of the total onion production of the country. Multiplier onion or aggregatum onion (Allium cepa var. 
aggregatum L.) is one of the most important commercial vegetable crops grown in South India especially in Tamil Nadu and Karnataka. The aggregatum onion accounts for nearly 75 percent of the total onion produced in Tamil Nadu with an average productivity of 12 tonnes per hectare (Pugalendhi et al., 2020).

The aggregatum onion produces many number of smaller sized bulbs in cluster form and mostly preferred for their tolerance to pest and diseases and longest storage life than common onion (Brewster, 2008, Anbukkarasi et al., 2012, Anbukkarasi et al., 2013, Pugalendhi et al., 2011a, Pugalendhi et al., $2011 b$,). Onion possess Quercetin and volatile sulfur compounds which helps in anemia, skin disorders, stomach cancer, eye infection, prevent asthma attack (Ahmed and Bassuorry, 2009 and Lanzotti et al., 2012). The bulb provides $2.0 \mathrm{~g}$ protein, $72 \mathrm{mg}$ calcium and 54 mg phosphorus. It also contains vitamins viz., thiamine, riboflavin and niacin and is used for its medicinal value especially in the case of heart problems (Mettananda and Fordham, 2001 and Shabina et al., 2020). Onions contain phenolics and flavonoids that have potential antiinflammatory, anticholesterol, anticancer and antioxidant properties. Onion is highly nutritional and it is used for lowering the toxigenicity of oils. It also shows chemo-preventive effects and lowers down the risk of effect on gastric cancer

The aggregatum onion cv. CO On 5 has got better market preference because of its size and appealing attractive pink colour. In India, presently about 35 to 40 per cent of the onion is estimated to be lost as post-harvest losses during various operations including handling and storage. In general, the losses due to reduction in weight, sprouting and rotting (decay) were found to be 20 to 25,4 to 5 and 10 to 12 per cent respectively (Pandey, 1989; Anon., 1994). The bulb respires and transpires continuously resulting in high weight loss and becomes susceptible to various diseases and spoilage due to inappropriate packaging. Adequate and proper packaging protects the bulb from physical (firmness), physiological (weight) and pathological (decay) deterioration.

One of the major problems confronted by small onion growers is the post-harvest losses due to lack of proper storage structures or lack of suitable field techniques to prevent or minimize these losses during storage. Postharvest losses mainly manifest through weight loss, sprouting, rooting and rotting of the bulbs etc. Pre-harvest sprays using growth regulator coupled with fungicide were tried in small onion bulbs in order to study their effects in the post-harvest physiology of the bulbs. Use of a proper structure is also one of the methods by which the post-harvest losses can be minimized. In Tamil Nadu, the onion growers are using a local structure called 'pattarai' in which the onions may be stored for a maximum period of four months. The cost of the 'pattarai' is comparatively very cheap but the post-harvest losses are considerable, the shelf life is very short and the structure cannot be reused. With this background, the present investigation was carried out to standardize the appropriate packing and storage techniques for enhancing quality and shelf life of onion cv. CO On 5.

\section{Materials and Methods}

The present investigations were carried out at the College Orchard, Tamil Nadu Agricultural University, Coimbatore. In addition to regular cultivation practices, Cycocel @ 200 ppm + Carbendazim@1000 ppm at 30 days before harvest was sprayed and the bulbs were harvested with $2.0 \mathrm{~cm}$ neck length were used in storage studies for three months. At the end of curing period, a composite sample of five $\mathrm{kg}$ bulbs were packed in different packing 
materials viz., 40kg open mesh jute bags weighing $250 \mathrm{~g}, 40 \mathrm{~kg}$ open mesh nylon net bags weighing $60 \mathrm{~g}$, baskets made up with split bamboo sticks $(20 \times 15 \times 25 \mathrm{~cm})$ of $5 \mathrm{~kg}$ capacity, perforated plastic crates $(50 \times 30 \times$ $28 \mathrm{~cm}$ ) of $15 \mathrm{~kg}$ capacity and the bulbs were stored in two methods i.e., low cost ventilation storage (constructed as per the specifications given by Tripathi and Lawande, 2005) and two different cold storage environments viz., $15^{\circ} \mathrm{C}$ with $60 \% \mathrm{RH}$ and $20^{\circ} \mathrm{C}$ with $80 \% \mathrm{RH}$. The details of the treatments are given below.

Design: Factor completely randomized design

Factor A - Type of packing and storage (T)

Factor B - Period of storage (P)

\section{Factor A. Treatments}

\begin{tabular}{|l|l|}
\hline $\mathrm{T}_{1-}$ & Packing in jute bags and kept in room temperature \\
\hline $\mathrm{T}_{2-}$ & Packing in nylon net bags and kept in room temperature \\
\hline $\mathrm{T}_{3-}$ & Packing in perforated plastic crates and kept in room temperature \\
\hline $\mathrm{T}_{4-}$ & Packing in bamboo baskets and kept in room temperature \\
\hline $\mathrm{T}_{5}-$ & Spreading of bulbs in ventilated storage \\
\hline $\mathrm{T}_{6}-$ & Spreading of bulbs in cold storage at $15^{\circ} \mathrm{C}$ \\
\hline $\mathrm{T}_{7-}$ & Spreading of bulbs in cold storage at $20^{\circ} \mathrm{C}$ \\
\hline $\mathrm{T} 8-$ & Spreading of bulbs in room temperature (Control) \\
\hline
\end{tabular}

\section{Factor B - Period of storage (P)}

$\mathrm{P}_{1^{-}} 30$ days after storage; $\quad \mathrm{P}_{2^{-}} \quad 60$ days after storage; $\mathrm{P}_{3^{-}} 90$ days after storage

Replications: Three Initial observations were recorded before imposing treatments and the storage observations were recorded at 30, 60 and 90 days after storage (monthly intervals). The bio-chemical parameters viz., total soluble solids (hand refractometer technique), ascorbic acid (AOAC, 1975), soluble protein (Lowery et al., 1971), sulphur (Chopra and Kanwar, 1991), pyruvic acid and total phenols (Bray and Thorpe, 1954) were studied. The enzyme activities viz., peroxidase (Srivastava, 1987), polyphenol oxidase (Mayer et al., 1965) and phenylalanine ammonia lyase activity (Dickerson et al., 1984) were analyzed.

The physiological parameters viz., physiological loss in weight (PLW), sprouting loss, rotting loss, total loss (PLW + sprouting loss+ rotting loss) also calculated as per the standard procedures. The per cent marketable bulbs were calculated by adopting the following formula.

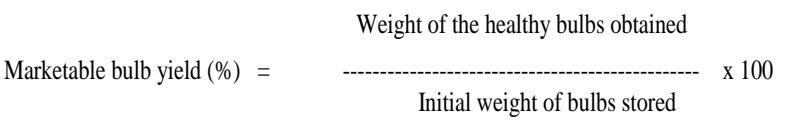

The rooted bulbs from the storage room were taken out, the weight was recorded and rooting loss was worked out accordingly.

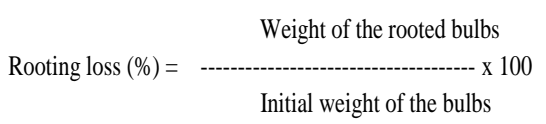

The data obtained from the present investigation were subjected to statistical scrutiny by adopting the standard procedure of Panse and Sukhatme (1985).

\section{Results and Discussion}

Onion bulbs in storage generally undergo loss in weight owing to physiological changes like 
sprouting, rooting, rotting and desiccation, nutritional and other bio-chemical changes.

\section{Physiological changes}

The lowest physiological loss in weight (5.18 per cent), sprouting (0.62 per cent), rotting (0.64 per cent), rooting ( 0.00 per cent $)$, total loss (8.19 per cent) and the highest marketable bulb (81.33\%) was observed in $\mathrm{T}_{5}$ (Spreading of bulbs in ventilated storage). Physiological loss in weight, rotting rooting and total loss was increased during the storage period. The least physiological loss in weight (6.76 per cent), rotting ( 0.04 per cent), rooting (3.63 per cent), total loss (13.52 per cent) and also the highest marketable bulbs $(73.85 \%)$ was recorded in $\mathrm{P}_{1}$ (30 days after storage). As the storage period increased from 30 to 90 days, sprouting also increased, except in cold storage treatments. In cold storage 90 per cent sprouting was obtained 30 days after storage. The least sprouting was registered in $\mathrm{P}_{3}(90$ days after storage) recording values of 1.50 per cent (Table 1, $2 \& 3$ ). Interaction between treatments and period of storage showed that, the minimum value of physiological loss in weight ( 2.72 per cent), sprouting $(0.15$ per cent $)$, rotting ( 0.00 per cent $)$, rooting $(0.00$ per cent), total loss (4.17 per cent) and also maximum marketable bulbs (88.12 and 90.95 $\%$ ) were recorded in interaction of $\mathrm{T}_{5} \mathrm{P}_{1}$ (Spreading of bulbs in ventilated storage for 30 days). In the present study, weight loss in bulbs was found to increase with increase in the storage period. Palaniswamy (1980) and Vincent (1980) in small onion also observed a similar phenomenon in storage.

In the present investigation, the bulbs stored in low cost bottom ventilated structure recorded the lowest physiological loss in weight, which is in accordance with the findings of Kopec (1963). This might be due to proper aeration, causing decreased temperature and humidity level inside the structure.
The dormant buds enclosed in the fleshy scales start sprouting in storage and earlier the sprouting in storage, the more is the loss to the growers as the sprouted bulbs have lesser weight than the non-sprouted ones. In the present study, the effect of various treatments on the sprouting of onion bulbs in storage showed that the bulbs started sprouting only after one month of storage. In the present finding, the bulbs kept under low cost bottom ventilated storage structure recorded less sprouting. The results of the present investigation are in conformity with the findings of Singh and Dhankhar (1995) and Kukanoor et al., (2005).

Rotting of onion bulbs is normally met during storage due to the pathogens, viz., Botrytis allii and Pseudomonas alliicola causing neck rot and soft rot developed due to excess humidity in the storage environment and mechanical damage during handling cause considerable loss to the growers. In the present investigation, pre-harvest sprayed bulbs stored in low cost bottom ventilated structure recorded reduced the rotting incidence. This might be due to good aeration and less humidity and optimum temperature inside the structure when compared to outside environment. Similar views were expressed by Shanthi and Balakrishnan (1989) and Waskar et al., (2004). The storage losses in onion bulbs are due to the triggering of metabolic process. Initiation of rooting is the triggering agent which results in depletion of stored food materials which are used as substrates for growth and development of the shoot initials. So, for proper storage of onion bulbs, the metabolic processes have to be blocked. In the present investigation, preharvest sprayed bulbs stored in low cost bottom ventilated structure showed lowest rooting. This might be due to less humidity inside the storage structure when compared to outer region. Higher levels of humidity enhanced the rooting (Jones and Mann, 1963). 
Table.1 Effect of packing and storage methods on physiological loss in weight (\%) and sprouting (\%) content in onion

\begin{tabular}{|c|c|c|c|c|c|c|c|c|}
\hline \multirow[t]{2}{*}{ Treatments } & \multicolumn{4}{|c|}{ Physiological loss in weight $(\%)$} & \multicolumn{4}{|c|}{ Sprouting (\%) } \\
\hline & $\mathbf{P}_{1}$ & $\mathbf{P}_{2}$ & $\mathbf{P}_{3}$ & Mean & $\mathbf{P}_{1}$ & $\mathbf{P}_{2}$ & $\mathbf{P}_{3}$ & Mean \\
\hline $\mathbf{T}_{1}$ & $5.33(13.35)$ & $7.53(15.93)$ & $13.75(21.77)$ & $8.87(17.02)$ & $0.27(2.56)$ & $0.83(5.24)$ & $1.30(6.55)$ & $0.80(4.78)$ \\
\hline $\mathbf{T}_{2}$ & $4.00(11.54)$ & $5.72(13.83)$ & $11.83(20.12)$ & $7.18(15.16)$ & $0.25(2.87)$ & $0.75(4.97)$ & $1.23(6.38)$ & $0.74(4.74)$ \\
\hline $\mathbf{T}_{3}$ & $3.55(10.86)$ & $4.67(12.48)$ & $10.52(18.91)$ & $6.25(14.08)$ & $0.20(2.56)$ & $0.70(4.80)$ & $1.15(6.16)$ & $0.68(4.51)$ \\
\hline $\mathbf{T}_{4}$ & $6.95(15.29)$ & $8.92(17.37)$ & $15.70(23.34)$ & $10.52(18.67)$ & $0.35(3.39)$ & $1.00(5.74)$ & $1.32(6.59)$ & $0.89(5.24)$ \\
\hline $\mathbf{T}_{5}$ & $2.72(9.49)$ & $3.00(11.54)$ & $9.83(18.28)$ & $5.18(13.10)$ & $0.15(2.22)$ & $0.55(4.25)$ & $1.15(6.16)$ & $0.62(4.21)$ \\
\hline$T_{6}$ & $11.98(20.25)$ & $13.87(21.86)$ & $21.10(27.35)$ & $15.65(23.15)$ & $16.00(23.58)$ & $12.00(20.27)$ & $2.27(8.59)$ & $10.09(17.48)$ \\
\hline $\mathbf{T}_{7}$ & $10.77(19.16)$ & $12.62(20.98)$ & $19.80(26.42)$ & $14.40(22.19)$ & $15.00(22.79)$ & $10.00(18.44)$ & $2.17(8.46)$ & $9.06(16.56)$ \\
\hline $\mathbf{T}_{8}$ & $8.78(17.24)$ & $10.78(19.17)$ & $17.47(24.70)$ & $12.34(20.37)$ & $0.50(4.05)$ & $1.10(6.02)$ & $1.40(6.80)$ & $1.00(5.62)$ \\
\hline \multirow[t]{2}{*}{ Mean } & $6.76(14.65)$ & $8.39(16.65)$ & $15.00(22.61)$ & 10.05 (17.97) & $4.09(8.00)$ & $3.74(8.72)$ & $1.50(6.96)$ & $3.11(7.89)$ \\
\hline & $\mathbf{T}$ & $\mathbf{P}$ & \multicolumn{2}{|c|}{$\mathbf{T} \times \mathbf{P}$} & $\mathbf{T}$ & $\mathbf{P}$ & \multicolumn{2}{|c|}{$\mathbf{T} \times \mathbf{P}$} \\
\hline SEd & 0.019 & 0.012 & \multicolumn{2}{|c|}{0.033} & 0.032 & 0.019 & \multicolumn{2}{|c|}{0.055} \\
\hline CD (0.05) & 0.038 & 0.023 & \multicolumn{2}{|c|}{0.065} & 0.063 & 0.038 & \multicolumn{2}{|c|}{0.109} \\
\hline
\end{tabular}

Table.2 Effect of packing and storage methods on rotting (\%) and rooting (\%) in onion

\begin{tabular}{|c|c|c|c|c|c|c|c|c|}
\hline \multirow[t]{2}{*}{ Treatments } & \multicolumn{4}{|c|}{ Rotting (\%) } & \multicolumn{4}{|c|}{ Rooting (\%) } \\
\hline & $\mathbf{P}_{1}$ & $\mathbf{P}_{2}$ & $\mathbf{P}_{3}$ & Mean & $\mathbf{P}_{1}$ & $\mathbf{P}_{2}$ & $\mathbf{P}_{3}$ & Mean \\
\hline $\mathbf{T}_{1}$ & $0.00(0.91)$ & $0.99(5.72)$ & $1.48(6.99)$ & $0.82(4.54)$ & $0.00(0.91)$ & $0.00(0.91)$ & $0.00(0.91)$ & $0.00(0.91)$ \\
\hline $\mathbf{T}_{2}$ & $0.00(0.91)$ & $0.96(5.63)$ & $1.35(6.67)$ & $0.77(4.40)$ & $0.00(0.91)$ & $0.00(0.91)$ & $0.00(0.91)$ & $0.00(0.91)$ \\
\hline $\mathbf{T}_{\mathbf{3}}$ & $0.00(0.91)$ & $0.90(5.44)$ & $1.33(6.63)$ & $0.74(4.33)$ & $0.00(0.91)$ & $0.00(0.91)$ & $0.00(0.91)$ & $0.00(0.91)$ \\
\hline $\mathbf{T}_{4}$ & $0.00(0.91)$ & $1.03(5.83)$ & $1.72(7.53)$ & $0.92(4.76)$ & $0.00(0.91)$ & $0.00(0.91)$ & $0.00(0.91)$ & $0.00(0.91)$ \\
\hline $\mathbf{T}_{5}$ & $0.00(0.91)$ & $0.85(5.29)$ & $1.08(5.97)$ & $0.64(4.06)$ & $0.00(0.91)$ & $0.00(0.91)$ & $0.00(0.91)$ & $0.00(0.91)$ \\
\hline$T_{6}$ & $0.12(1.95)$ & $1.12(6.07)$ & $2.05(8.23)$ & $1.10(5.42)$ & $13.00(21.13)$ & $16.67(24.10)$ & $31.33(34.04)$ & $20.33(26.42)$ \\
\hline $\mathbf{T}_{7}$ & $0.05(1.28)$ & $1.05(5.88)$ & $1.95(8.03)$ & $1.02(5.06)$ & $16.00(23.58)$ & $20.83(27.16)$ & $32.67(34.86)$ & 23.17 \\
\hline $\mathbf{T}_{8}$ & $0.12(1.95)$ & $1.22(6.33)$ & $2.07(8.27)$ & $1.14(5.52)$ & $0.00(0.91)$ & $0.00(0.91)$ & $0.00(0.91)$ & $0.00(0.91)$ \\
\hline \multirow[t]{2}{*}{ Mean } & $0.04(1.22)$ & $1.02(5.77)$ & 1.57 (7.29) & $0.87(4.76)$ & $3.63(6.27)$ & $4.69(7.09)$ & $8.00(9.30)$ & $5.44(7.55)$ \\
\hline & $\mathbf{T}$ & $\mathbf{P}$ & \multicolumn{2}{|c|}{$\mathbf{T} \times \mathbf{P}$} & $\mathbf{T}$ & $\mathbf{P}$ & \multicolumn{2}{|c|}{$\mathbf{T} \times \mathbf{P}$} \\
\hline SEd & 0.021 & 0.013 & \multicolumn{2}{|c|}{0.036} & 0.047 & 0.029 & \multicolumn{2}{|c|}{0.082} \\
\hline CD (0.05) & 0.041 & 0.025 & \multicolumn{2}{|c|}{0.071} & 0.094 & 0.058 & \multicolumn{2}{|c|}{0.163} \\
\hline
\end{tabular}


Table.3 Effect of packing and storage methods on total loss (\%) and marketable bulbs (\%) in onion

\begin{tabular}{|c|c|c|c|c|c|c|c|c|}
\hline \multirow[t]{2}{*}{ Treatments } & \multicolumn{4}{|c|}{ Total loss $(\%)$} & \multicolumn{4}{|c|}{ Marketable bulbs (\%) } \\
\hline & $\mathbf{P}_{1}$ & $\mathbf{P}_{2}$ & $\mathbf{P}_{3}$ & Mean & $\mathbf{P}_{1}$ & $\mathbf{P}_{2}$ & $\mathbf{P}_{3}$ & Mean \\
\hline $\mathbf{T}_{1}$ & $5.60(13.69)$ & $9.36(17.81)$ & $16.53(23.99)$ & $10.50(18.50)$ & $85.12(67.31)$ & $73.52(59.03)$ & $63.70(52.96)$ & $74.11(59.77)$ \\
\hline $\mathbf{T}_{2}$ & $4.25(11.90)$ & $7.43(15.82)$ & $14.42(22.32)$ & $8.70(16.68)$ & $85.78(67.85)$ & $73.85(59.25)$ & $70.77(57.27)$ & $76.80(61.46)$ \\
\hline $\mathbf{T}_{3}$ & $3.75(11.17)$ & $6.27(14.50)$ & $12.98(21.12)$ & $7.67(15.60)$ & $86.05(68.07)$ & $77.57(61.73)$ & $72.23(58.20)$ & $78.62(62.67)$ \\
\hline $\mathbf{T}_{4}$ & $7.30(15.68)$ & $10.95(19.32)$ & $18.73(25.65)$ & $12.33(20.22)$ & $84.00(66.42)$ & $72.67(58.48)$ & $67.85(55.46)$ & $74.84(60.12)$ \\
\hline $\mathbf{T}_{5}$ & $2.87(9.75)$ & $5.40(13.44)$ & $12.07(20.33)$ & $6.78(14.51)$ & $90.95(72.49)$ & 79.07 (62.77) & $73.98(59.33)$ & $81.33(64.86)$ \\
\hline$T_{6}$ & $28.10(32.01)$ & $26.98(31.30)$ & $25.38(30.25)$ & $26.82(31.19)$ & $35.78(36.74)$ & $30.52(33.53)$ & $22.77(28.50)$ & $29.69(32.92)$ \\
\hline $\mathbf{T}_{7}$ & $25.82(30.54)$ & $23.87(29.25)$ & $23.92(29.28)$ & 24.54 (29.69) & $38.97(38.63)$ & $34.03(35.69)$ & $28.62(32.34)$ & $33.87(35.55)$ \\
\hline $\mathbf{T}_{8}$ & $9.40(17.85)$ & $13.10(21.22)$ & $20.93(27.23)$ & $14.48(22.10)$ & $84.13(66.53)$ & $68.97(56.15)$ & $61.78(51.82)$ & $71.63(58.17)$ \\
\hline \multirow[t]{2}{*}{ Mean } & $10.89(17.82)$ & $12.92(20.33)$ & $18.12(25.02)$ & $13.98(21.06)$ & $73.85(60.51)$ & $63.78(53.33)$ & $57.71(49.49)$ & $65.11(54.44)$ \\
\hline & $\mathbf{T}$ & $\mathbf{P}$ & \multicolumn{2}{|c|}{$\mathbf{T} \times \mathbf{P}$} & $\mathbf{T}$ & $\mathbf{P}$ & \multicolumn{2}{|c|}{$\mathbf{T} \times \mathbf{P}$} \\
\hline SEd & 0.029 & 0.018 & \multicolumn{2}{|c|}{0.050} & 0.036 & 0.022 & \multicolumn{2}{|c|}{0.063} \\
\hline CD (0.05) & 0.057 & 0.035 & \multicolumn{2}{|c|}{0.099} & 0.072 & 0.044 & \multicolumn{2}{|c|}{0.125} \\
\hline
\end{tabular}

Table.4 Effect of packing and storage methods on TSS $\left({ }^{\circ}\right.$ Brix $)$ and ascorbic acid (mg $\left.100 \mathrm{~g}^{-1}\right)$ content in onion

\begin{tabular}{|c|c|c|c|c|c|c|c|c|}
\hline \multirow[t]{2}{*}{ Treatments } & \multicolumn{4}{|c|}{ TSS content $\left({ }^{\circ} \mathrm{Brix}\right)$} & \multicolumn{4}{|c|}{ Ascorbic acid content (mg $\left.100 \mathrm{~g}^{-1}\right)$} \\
\hline & $\mathbf{P}_{1}$ & $\mathbf{P}_{2}$ & $\mathbf{P}_{\mathbf{3}}$ & Mean & $\mathbf{P}_{1}$ & $\mathbf{P}_{2}$ & $\mathbf{P}_{3}$ & Mean \\
\hline $\mathbf{T}_{1}$ & 14.77 & 16.52 & 18.55 & 16.61 & 9.75 & 9.67 & 9.48 & 9.63 \\
\hline $\mathbf{T}_{2}$ & 14.82 & 16.58 & 18.62 & 16.67 & 9.96 & 9.83 & 9.75 & 9.85 \\
\hline $\mathbf{T}_{3}$ & 15.02 & 16.78 & 18.80 & 16.87 & 10.12 & 9.95 & 9.82 & 9.96 \\
\hline $\mathbf{T}_{4}$ & 14.52 & 16.38 & 18.38 & 16.43 & 9.68 & 9.50 & 9.07 & 9.42 \\
\hline $\mathbf{T}_{5}$ & 15.17 & 17.15 & 19.33 & 17.22 & 10.60 & 10.12 & 9.99 & 10.24 \\
\hline$T_{6}$ & 14.13 & 16.15 & 18.27 & 16.18 & 9.17 & 9.08 & 8.93 & 9.06 \\
\hline $\mathbf{T}_{7}$ & 14.18 & 16.20 & 18.32 & 16.23 & 9.47 & 9.25 & 9.00 & 9.24 \\
\hline $\mathbf{T}_{8}$ & 14.03 & 16.08 & 18.03 & 16.05 & 8.90 & 8.68 & 8.47 & 8.68 \\
\hline \multirow[t]{2}{*}{ Mean } & 14.58 & 16.48 & 18.54 & 16.53 & 9.71 & 9.51 & 9.31 & 9.51 \\
\hline & $\mathbf{T}$ & $\mathbf{P}$ & \multicolumn{2}{|c|}{$\mathbf{T} \times \mathbf{P}$} & $\mathbf{T}$ & $\mathbf{P}$ & \multicolumn{2}{|c|}{$\mathbf{T} \times \mathbf{P}$} \\
\hline SEd & 0.007 & 0.005 & \multicolumn{2}{|c|}{0.013} & 0.051 & 0.031 & \multicolumn{2}{|c|}{0.087} \\
\hline CD (0.05) & 0.015 & 0.009 & \multicolumn{2}{|c|}{0.026} & 0.100 & 0.061 & \multicolumn{2}{|c|}{0.174} \\
\hline
\end{tabular}


Table.5 Effect of packing and storage methods on pyruvic acid content $\left(\mu \mathrm{mol} \mathrm{g}^{-1}\right)$ and sulphur content $(\%)$ in onion

\begin{tabular}{|c|c|c|c|c|c|c|c|c|}
\hline \multirow[t]{2}{*}{ Treatments } & \multicolumn{4}{|c|}{ Pyruvic acid content $\left(\mu \mathrm{mol} \mathrm{g}^{-1}\right)$} & \multicolumn{4}{|c|}{ Sulphur content $(\%)$} \\
\hline & $\mathbf{P}_{1}$ & $\mathbf{P}_{2}$ & $\mathbf{P}_{3}$ & Mean & $\mathbf{P}_{1}$ & $\mathbf{P}_{2}$ & $\mathbf{P}_{3}$ & Mean \\
\hline $\mathbf{T}_{1}$ & 2.48 & 2.44 & 2.35 & 2.42 & 0.622 & 0.692 & 0.745 & 0.686 \\
\hline $\mathbf{T}_{2}$ & 2.50 & 2.46 & 2.37 & 2.44 & 0.630 & 0.695 & 0.755 & 0.693 \\
\hline $\mathbf{T}_{3}$ & 2.59 & 2.50 & 2.40 & 2.50 & 0.635 & 0.698 & 0.765 & 0.699 \\
\hline $\mathbf{T}_{4}$ & 2.44 & 2.40 & 2.34 & 2.39 & 0.617 & 0.685 & 0.740 & 0.681 \\
\hline $\mathbf{T}_{5}$ & 2.60 & 2.54 & 2.44 & 2.53 & 0.643 & 0.700 & 0.768 & 0.704 \\
\hline $\mathbf{T}_{6}$ & 2.39 & 2.36 & 2.28 & 2.34 & 0.596 & 0.673 & 0.688 & 0.652 \\
\hline $\mathbf{T}_{8}$ & 2.35 & 2.34 & 2.25 & 2.31 & 0.610 & 0.680 & 0.713 & 0.668 \\
\hline \multirow[t]{2}{*}{ Mean } & 2.47 & 2.43 & 2.34 & 2.41 & 0.620 & 0.687 & 0.733 & 0.680 \\
\hline & $\mathbf{T}$ & $\mathbf{P}$ & \multicolumn{2}{|c|}{$\mathbf{T} \times \mathbf{P}$} & $\mathbf{T}$ & $\mathbf{P}$ & \multicolumn{2}{|c|}{$\mathbf{T} \times \mathbf{P}$} \\
\hline SEd & 0.004 & 0.002 & \multicolumn{2}{|c|}{0.006} & 0.005 & 0.000 & \multicolumn{2}{|c|}{0.001} \\
\hline CD (0.05) & 0.007 & 0.004 & \multicolumn{2}{|c|}{0.012} & 0.001 & 0.001 & \multicolumn{2}{|c|}{0.002} \\
\hline
\end{tabular}

Table.6 Effect of packing and storage methods on phenylalanine ammonia lyase activity $\left(\mu \mathrm{g} \mathrm{ml}^{-1} \mathrm{~min}^{-1}\right)$ and peroxidase activity (absorbance $\mathrm{g}^{-1} \mathrm{~min}^{-1}$ ) in onion

\begin{tabular}{|c|c|c|c|c|c|c|c|c|}
\hline \multirow[t]{2}{*}{ Treatments } & \multicolumn{4}{|c|}{ Phenylalanine ammonia lyase activity $\left(\mu \mathrm{g} \mathrm{m} \mathrm{m}^{-1} \mathrm{~min}^{-1}\right)$} & \multicolumn{4}{|c|}{ Peroxidase activity (absorbance $\mathrm{g}^{-1} \mathrm{~min}^{-1}$ ) } \\
\hline & $\mathbf{P}_{1}$ & $\mathbf{P}_{2}$ & $\mathbf{P}_{3}$ & Mean & $\mathbf{P}_{1}$ & $\mathbf{P}_{2}$ & $\mathbf{P}_{3}$ & Mean \\
\hline $\mathbf{T}_{1}$ & 5.97 & 5.00 & 4.20 & 5.06 & 1.831 & 1.662 & 1.642 & 1.712 \\
\hline $\mathbf{T}_{2}$ & 6.30 & 5.81 & 5.18 & 5.76 & 1.833 & 1.689 & 1.670 & 1.731 \\
\hline $\mathbf{T}_{3}$ & 7.20 & 6.30 & 5.64 & 6.38 & 1.848 & 1.708 & 1.683 & 1.746 \\
\hline $\mathbf{T}_{4}$ & 5.30 & 4.80 & 4.10 & 4.73 & 1.819 & 1.472 & 1.255 & 1.515 \\
\hline $\mathbf{T}_{5}$ & 7.75 & 6.80 & 6.04 & 6.86 & 1.895 & 1.818 & 1.763 & 1.825 \\
\hline $\mathbf{T}_{6}$ & 3.46 & 2.80 & 2.00 & 2.75 & 1.312 & 0.821 & 0.647 & 0.927 \\
\hline $\mathbf{T}_{8}$ & 5.15 & 4.15 & 3.40 & 4.23 & 1.810 & 1.453 & 1.240 & 1.501 \\
\hline \multirow[t]{2}{*}{ Mean } & 5.75 & 4.92 & 4.18 & 4.95 & 1.724 & 1.461 & 1.335 & 1.506 \\
\hline & $\mathbf{T}$ & $\mathbf{P}$ & \multicolumn{2}{|c|}{$\mathbf{T} \times \mathbf{P}$} & $\mathbf{T}$ & $\mathbf{P}$ & \multicolumn{2}{|c|}{$\mathbf{T} \times \mathbf{P}$} \\
\hline SEd & 0.003 & 0.002 & \multicolumn{2}{|c|}{0.005} & 0.002 & 0.001 & \multicolumn{2}{|c|}{0.003} \\
\hline CD (0.05) & 0.006 & 0.004 & \multicolumn{2}{|c|}{0.010} & 0.003 & 0.002 & \multicolumn{2}{|c|}{0.005} \\
\hline
\end{tabular}


Table.7 Effect of packing and storage methods on polyphenol oxidase activity (absorbance $\left.\mathrm{g}^{-1} \mathrm{~min}^{-1}\right)$ and total phenolics $\left(\mu \mathrm{g} \mathrm{g}^{-1}\right)$ in onion

\begin{tabular}{|c|c|c|c|c|c|c|c|c|}
\hline \multirow[t]{2}{*}{ Treatments } & \multicolumn{4}{|c|}{ Polyphenol oxidase activity (absorbance $\mathrm{g}^{-1} \mathrm{~min}^{-1}$ ) } & \multicolumn{4}{|c|}{ Total phenolics $\left(\mu \mathrm{g} \mathrm{g}^{-1}\right)$} \\
\hline & $\mathbf{P}_{1}$ & $\mathbf{P}_{2}$ & $\mathbf{P}_{\mathbf{3}}$ & Mean & $\mathbf{P}_{1}$ & $\mathbf{P}_{2}$ & $\mathbf{P}_{3}$ & Mean \\
\hline $\mathbf{T}_{1}$ & 1.345 & 1.194 & 1.151 & 1.230 & 622.33 & 608.67 & 610.00 & 613.67 \\
\hline $\mathbf{T}_{2}$ & 1.362 & 1.247 & 1.204 & 1.271 & 631.67 & 611.67 & 613.67 & 619.00 \\
\hline $\mathbf{T}_{\mathbf{3}}$ & 1.369 & 1.280 & 1.216 & 1.288 & 635.33 & 619.00 & 615.67 & 623.33 \\
\hline $\mathbf{T}_{4}$ & 1.317 & 1.058 & 0.909 & 1.095 & 619.00 & 605.67 & 606.00 & 610.22 \\
\hline $\mathbf{T}_{5}$ & 1.382 & 1.323 & 1.258 & 1.321 & 638.33 & 622.67 & 615.67 & 625.56 \\
\hline $\mathbf{T}_{6}$ & 0.868 & 0.707 & 0.571 & 0.715 & 531.00 & 531.67 & 516.00 & 526.22 \\
\hline $\mathbf{T}_{7}$ & 0.985 & 0.807 & 0.611 & 0.801 & 558.33 & 540.67 & 528.00 & 542.33 \\
\hline $\mathbf{T}_{\mathbf{8}}$ & 1.310 & 1.031 & 0.789 & 1.043 & 620.00 & 598.33 & 582.67 & 600.33 \\
\hline Mean & 1.242 & 1.081 & 0.964 & 1.096 & 607.00 & 592.29 & 585.96 & $\mathbf{5 9 5 . 0 8}$ \\
\hline & $\mathbf{T}$ & $\mathbf{P}$ & \multicolumn{2}{|c|}{$\mathbf{T} \times \mathbf{P}$} & $\mathbf{T}$ & $\mathbf{P}$ & \multicolumn{2}{|c|}{$\mathbf{T} \times \mathbf{P}$} \\
\hline SEd & 0.002 & 0.001 & \multicolumn{2}{|c|}{0.004} & 0.455 & 0.278 & \multicolumn{2}{|c|}{0.788} \\
\hline CD (0.05) & 0.004 & 0.002 & \multicolumn{2}{|c|}{0.007} & 0.902 & 0.553 & \multicolumn{2}{|c|}{1.563} \\
\hline
\end{tabular}


In the present investigation, pre harvest sprayed onion bulbs harvested with $2 \mathrm{~cm}$ neck bulbs stored in low cost bottom ventilated structure recorded the highest per cent of marketable bulbs compared to other treatments. This may be attributed to minimum physiological loss in weight, rotting and sprouting in these treatments (Blanco and Oliveira, 1971).

\section{Biochemical changes}

The highest TSS (16.83 ${ }^{\circ}$ brix), ascorbic acid $\left(9.59 \mathrm{mg} 100 \mathrm{~g}^{-1}\right)$, pyruvic acid $\left(2.48 \mu \mathrm{mol} \mathrm{g}{ }^{-1}\right)$, sulphur (0.694 per cent), total phenolics content $\left(621.08 \mu \mathrm{g} \quad \mathrm{g}^{-1}\right)$, phenylalanine ammonia lyase activity $\left(6.44 \mu \mathrm{g} \mathrm{ml}^{-1} \mathrm{~min}^{-1}\right)$, peroxidase activity (1.789 absorbance $\mathrm{g}^{-1} \mathrm{~min}^{-1}$ ) and polyphenol oxidase activity (1.237 absorbance $\mathrm{g}^{-1} \mathrm{~min}^{-1}$ ) was recorded in $\mathrm{T}_{5}$ (Spreading of bulbs in ventilation storage). The storage period increased from 30 to 90 days, the ascorbic acid, pyruvic acid, total phenolic content, phenylalanine ammonia lyase activity, peroxidase and polyphenol oxidase activity decreased. The maximum ascorbic acid $\left(8.85 \mathrm{mg} 100 \mathrm{~g}^{-1}\right)$, pyruvic acid

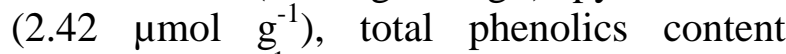
(600.01 $\mu \mathrm{g} \mathrm{g}^{-1}$ ), phenylalanine ammonia lyase activity $\left(5.41 \mu \mathrm{g} \mathrm{ml}^{-1} \mathrm{~min}^{-1}\right)$, peroxidase activity (1.661 absorbance $\mathrm{g}^{-1} \mathrm{~min}^{-1}$ ) and polyphenol oxidase activity $\quad(1.115$ absorbance $\mathrm{g}^{-1} \mathrm{~min}^{-1}$ ) was registered in $\mathrm{P}_{1}$ (30 days after storage). As the storage period increased from 30 to 90 days, the TSS and sulphur content was increased. The highest TSS (17.90 ${ }^{\circ}$ brix) and sulphur content (0.726 per cent) was noticed in $\mathrm{P}_{3}$ (90 days after storage). Interaction between treatments and period of storage showed that the maximum ascorbic acid (9.95 mg $100 \mathrm{~g}^{-1}$ ), pyruvic acid

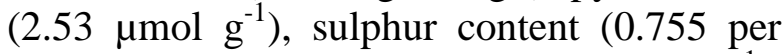
cent), total phenolics content $\left(631.89 \mu \mathrm{g} \mathrm{g}^{-1}\right)$, phenylalanine ammonia lyase activity (7.23 $\left.\mu \mathrm{g} \mathrm{ml}^{-1} \mathrm{~min}^{-1}\right)$, peroxidase activity (1.830 absorbance $\mathrm{g}^{-1} \mathrm{~min}^{-1}$ ) and polyphenol oxidase activity (1.267 and 1.273 absorbance $\mathrm{g}^{-1} \mathrm{~min}^{-1}$ ) was observed in $\mathrm{T}_{5} \mathrm{P}_{1}$ (Spreading of bulbs in ventilated storage +30 days after storage) and also the highest TSS content of $18.49^{\circ}$ brix was noticed in $\mathrm{T}_{5} \mathrm{P}_{3}$ (Spreading of bulbs in ventilated storage +90 days after storage) (Table 4, 5, $6 \&$ 7).

In the present study, the quality parameters of bulbs improved gradually in all the treatments during storage. It might be due to gradual decrease in moisture content of the bulbs during storage and consequent increase in concentration of nutrients present in the tissue. The respiratory rate would be low due to loss of moisture causing increased content of total sugars. These findings are in agreement with the findings of Misra and Pande (1979).

The TSS contributes major part in turgidity maintenance of bulbs and which is governed by the osmo-regulatory mechanism. Increased TSS content evidently shows that the stored food materials undergo either partial or complete hydrolysis and provide substrates for respiration. In the present study, the TSS content of the bulbs, in general, showed an increasing trend with increase in the storage period. This may be due to break down of complex polymers in to simple substances by hydrolytic enzymes (Deol, 1985). Similar observations were also recorded by Palaniswamy (1980).

In the present investigation, the ascorbic acid and pyruvic acid content of the bulbs, decreased gradually with the increase in storage period. This may be due to oxidation of L-ascorbic acid in to dehydro ascorbic acid by enzyme ascorbinase (Joshi and Roy, 1985). In the present study, pyruvic acid content decreased as the storage period increased. The results are also supported by the findings of Shock et al., (2004). The sulphur content of the bulbs gradually 
increased in all the treatments as the storage period advanced. In the present investigation, pre-harvest sprayed bulbs stored in low cost bottom ventilated structure registered higher sulphur content.

Further, pre-harvest sprayed bulbs stored in low cost bottom ventilated structure showed increased phenylalanine ammonia lyase, peroxidase and polyphenol oxidase activity. The enzymes indirectly reduced the sprouting percentage. Alternatively when the storage period was increased the enzyme activity decreased and increased the sprouting percentage.

The present study indicated that the bulbs stored under low cost bottom ventilated storage structure recorded lowest total phenol content with the increasing storage period. When the total phenol content was high, the enzyme activities were high. This would have induced resistance to pathogen by production of PR (Plant Resistance) proteins (Raskin, 1992).

In conclusion the pre harvest sprayed bulbs stored with $2.0 \mathrm{~cm}$ neck length in low cost bottom ventilated storage structure increased the shelf life of aggregatum onion cv. CO On 5 up to six months.

\section{References}

Ahmed, A. A. and Bassuony, N. I.. 2009. Importance of medical herbs in animal feeding. World J. Agric. Sci., 5: 456-465.

A.O.A.C. 1975. Official Methods of Analysis. $12^{\text {th }}$ edition Association of official analytical chemists. Washington D.C., U.S.A.

Anbukkarasi,V., Paramaguru, P., Pugalendhi, L., Ragupathi, N., Jeyakumar, P., 2013. Studies on pre and post-harvest treatments for extending shelf life in onion - A review.

Agric.

Reviews. 34 (4): 256-268.

Anbukkarasi,V., Paramaguru, P., Pugalendhi, L., Ragupathi, N., Jeyakumar, P. 2012. Temperature treatments on postharvest handling of small onion (Allium cepa L. var. aggregatum Don.). Madras Agric. Journal. 99(10/12): 673-676.

Anonymous, 1994. Post-harvest losses. Research digests. AICRP on postharvest technology (1980-1990). Gujarat Agric. University, Junagadh.

Blanco, H. G. and Oliveria, D. A. 1971. The effect of foliar applied growth regulators on the storage of onion bulbs. Arguivas do Institute Biologioc., 38(3): 89-94.

Bray, H. G. and Thorpe, N. U. 1954. Analysis of phenolic compounds of interest in metabolism. Math. Biochem. Anal., 9: 27-52.

Brewster, J. L. 2008. Onions and other Vegetable Alliums. (2nd ed.), CAB International, North America.

Chopra, S. L. and Kanwar, J. S. 1991. Analytical Agriculture chemistry. Kalyani publishers, New Delhi.

Deol, I. S. 1985. A study on chemical changes during the storage life of samara bahist mango (Mangifera indica L.). Punjab Hort. J., 25 (1- 4): 5-11.

Dickerson, D. P., S. F. Pascholati, A. E. Hagerman, L. G. Butler and R. L. Nicholson. 1984. phenylalanine ammonia lyase and hydroxyl cinnamate - CoA ligase in maize mesocotyls inoculated with Helminthosporium maidis (or) $H$. carborum. Physiol. Pl. Pathol., 25: 111-123.

Jones, H. A. and Mann, L .K. 1963. Onions and their allies. World crop series. Leonard Hill (Books) Limited, London.

Joshi, G. D. and Roy, S. K. 1985. Effect of 
integrated post-harvest handling on bio-chemical changes in alphonso mango fruits. Prog. Hort., 17(1): 5663.

Kopec, K. 1963. Chemical retardation of stored vegetables. Hort. Abstr., 34: 6679.

Kukanoor, L., Basavarajan and Rokhade, A. K. 2005. Effect of pre-harvest treatments and chemicals on postharvest quality and storage of onion. Asian. J. Hort., 2(1): 53-57.

Lanzotti, V. A. Romano, S. Lanzuise, G. Bonanomi and F. Scala, 2012. Antifungal saponins from bulbs of white onion Allium cepa L. Phytochemistry, 74: 133-139.

Lowery, O. H., Brought, N. T. R., Farrand L. A. and Randall, R.J. 1971. Protein measurement with folin phenol reagent. J. Biol. Chem., 193: 265-275.

Mayer, A. M., Haul, V., and Shaul, R. B. 1965. Assay of catechol oxidase, a critical comparison of method. Phytochem., 5: 783-789.

Mettananda, K. A., Fordham, R. 2001. The effects of plant size and leaf number on the bulbing of tropical short-day onion cultivars (Allium cepa L.) under controlled environments. J. Hortic. Sci., 14(5): 22-31.

Misra, R.S. and Pande, S.C. 1979. Biochemical changes in onion bulbs during storage. Punjab Hort. J., XIX (1 \&2): 86-88.

National Horticultural Board, 2019. Area and Production of Horticulture Crops - All India. New Delhi.

Palaniswamy, K.P. 1980. Studies on growth, development and storage of $\mathrm{CO}-2$ onion (Alliam cepa L. var. aggregatum Don.). Ph. D. Thesis, Department of Vegetable Crops, Tamil Nadu Agricultural University, Coimbatore.

Pandey, U.B. 1989. Problems in post-harvest handling of onion and current status of research work done by AADF in the field of post-harvest technology. AADF News Letter, 9(3 \& 4): 12-15.

Panse, V. G. and Sukhatme, P. V. 1985. The statistical methods for agricultural workers, ICAR Publications, New Delhi.

Pugalendhi, L., Sathiyamurty, V. A., Sumathi, T., Thangamani, C. 2011a. Weed management studies in onion. National Symposium on Alliums: Current Scenario and Emerging trends. 257.

Pugalendhi, L., Thangamani, C., Ragupathi, N. and Sumathi, T. 2011b. Studies on integrated nutrient management in onion (Allium cepa L.). Allium and Umbelliferae Improvement Newsletter. 21: 45-48.

Pugalendhi, L., K. S. Thingalmaniyan and Velmurugan, M. 2020. Evaluation of seed setting aggregatum onion (Allium cepa var. aggregatum) types under Tamil Nadu conditions. Int. J. Curr. Microbiol. App. Sci. 9(08): 3304-3310.

Raskin. 1992. Salicylate. A new plant hormone. Plant Physiol., 99: 799-803.

Shabina, S. Sharma, G. Irfan, M. A. 2020. A Review: Allium cepa aggregatum, characteristics, and properties. Gyan vihar. 6(1):1-6.

Shanthi, K. and Balakrishnan, R. 1989. Effect of nitrogen, spacing and maleic hydrazide on yield, nutrient uptake, quality and storage of MDU-1 onion. Indian J. Hort., 46(4): 490-495.

Sharma, H. G., Verma L .S., Jain V. and Tiwary, B. L. 1995. Effect of foliar spray of some growth regulators on growth and flowering of chrysanthemum var. Move-in. Carvin. Orissa J. Hort., 23(1\&2): 61-64.

Shock, C. C., Felbert E. B. G., and Saunders, L. D. 2004. Pungency of selected 
onion varieties before and after storage. Malheur experiment station. Annual report. Oregon State University Agricultural Experimental Station. Special report, 10555: 45-46.

Singh, J. and Dhankhar, B. S. 1995. Effect of pre-harvest chemical treatment on storage loss of onion. Adv. Horticulture and Forestry, 4: 119-126.

Srivastava, S. K. 1987. Peroxidase and polyphenol oxidase in Brassica juncea plants infected with Macrophomina phaseolina (Tassi) Goid and their implication in disease resistance. J. Phytopath., 120: 249254.

Tripathi, P. C. and K. E. Lawande. 2005. Low cost bottom ventilated onion storage structure. Technical Bulletin 12, NRCOG, Nasik, Maharashtra.

Vincent, R. S. 1980. Studies on the effect of different levels of nitrogen, phosphorus and potassium on $\mathrm{CO} .3$ onion (Allium cepa L. var. aggregatum Don.) M.Sc. (Ag.) Thesis, Department of Vegetable Crops, Tamil Nadu Agricultural University, Coimbatore.

Waskar, D. P., Gaikwad, R. S., Damame S. V. and Masalkar, S.D. 2004. Effect of pre-harvest sprays of growth regulators and fungicides on storage of onion. NHRDF News letter. XXIV (4):6-8.

\section{How to cite this article:}

Anbukkarasi, V., P. Paramaguru, L. Pugalendhi and Jayakumar, P. 2020. Effect of Packing and Storage Methods on Quality and Shelf Life of Onion (Allium cepa L. var. Aggregatum Don.). Int.J.Curr.Microbiol.App.Sci. 9(11): 611-622. doi: https://doi.org/10.20546/ijcmas.2020.911.075 